\title{
Measurements of pion production in eA with the CLAS detector
}

\author{
S. Manly and H. Lee ${ }^{1}$ \\ Department of Physics and Astronomy \\ University of Rochester \\ Rochester, NY 14627
}

\begin{abstract}
Preliminary results on semi-inclusive charged pion production in eA collisions at $\mathrm{E}_{\text {beam }}=5 \mathrm{GeV} / \mathrm{c}^{2}$ are presented. The data were collected using the CLAS detector, which is a multipurpose, large acceptance, magnetic spectrometer located in Hall B at the Thomas Jefferson National Accelerator Facility. Selected distributions in $\mathrm{W}, \mathrm{Q}^{2}, \mathrm{p}_{\pi}$, and $\theta_{\pi}$ are shown for data produced using deuterium, carbon, iron and lead targets. Preliminary comparisons with data simulated using the GENIE generator are made. The motivation for this work is to provide distributions useful for tuning the hadronic final state interaction models in order to reduce uncertainties in the results extracted from current and next-generation neutrino oscillation experiments.
\end{abstract}

Keywords: electron, neutrino, scattering, cross sections, Jefferson Laboratory

PACS: $25.30,13.15 .+\mathrm{g}$

\section{INTRODUCTION}

In order to improve the precision in current and next generation neutrino oscillation measurements[1], it is important to minimize systematic errors arising from nuclear models and the modeling of nuclear final state interactions of the produced hadrons. Electron scattering offers the opportunity to study nuclear effects in the hadron production in a system similar to what is seen with neutrinos and to tune the nuclear final-state interaction (FSI) portions of the neutrino interaction simulation code[2]. The aim of this work is to provide differential cross section data for pion production in electron scattering on several nuclear targets of interest to the neutrino community for this purpose. At this conference, disagreements between theory and experiment in the pion energy distribution have been shown, underscoring concerns about our understanding of FSI and the need for the measurements presented here[3].

\section{EXPERIMENT}

This analysis uses data from the CLAS experiment[5] at Thomas Jefferson National Accelerator Facility (JLAB)[4]. The data were taken during the "eg2" running period in 2003 and 2004[6]. During this time, an electron beam of $\sim 4-5 \mathrm{GeV}$ was incident on two nuclear targets simultaneously. One of those targets was cryogenic deuterium while the

\footnotetext{
${ }^{1}$ Representing the CLAS Collaboration.
} 
other was a solid target fabricated from either carbon, aluminum, iron, tin, or lead[8]. For the data used in this analysis, the beam energy was $5.014 \mathrm{GeV}$.

From a sample of over 4.8 billion interaction triggers, events with an electron and one detected charged pion were extracted. A number of selection criteria were invoked to insure only high quality, well-reconstructed events with the topology of interest were kept for continued analysis[7]. After all cuts approximately 34.6/6.1/9.3/3.1 million events with the electron interacting in the deuterium/carbon/iron/lead target were retained, respectively.

The large number of events in the data sample allows for the study of multi-differential cross sections which are, in principle, more powerful than integrated cross sections in constraining models of nuclear effects in these interactions. Cross sections were calculated using two variables that depend on leptonic information, $\mathrm{W}$ and $\mathrm{Q}^{2}$, and three variables that depend on pion information, $\mathrm{p}_{\pi}, \theta_{\pi}$ (the angle with respect to the beam direction) and the pion charge.

The data were corrected for acceptance and bin migration in a five-dimensional space using information from the Monte Carlo (see below). Radiative corrections were performed on the data using the ratio of cross sections calculated with and without radiative corrections determined differentially based on the leptonic variables, $\mathrm{W}$ and $\mathrm{Q}^{2}$, where the elastic contribution was removed (since we require a pion to be present)[9].

\section{SIMULATED EVENTS}

The use of simulated data in this work was essential for studies of acceptance, radiative corrections, and systematic errors. Simulated samples were created using the GENIE Monte Carlo generator package[10] with the eA mode enabled. Relative to the default GENIE used for neutrino interactions, the eA mode of GENIE uses charged lepton cross sections from Rein-Sehgal[11] and Bodek-Yang[12] and includes small modifications to account for the probe charge in the hadronization model and resonance event generation[13].

Events generated by GENIE were passed through the CLAS detector simulation (GSIM) and then processed through the same analysis chain used for the data. After processing, approximately 0.98/0.79/0.68/0.60 million interactions (with a produced charged pion) on the deuterium/carbon/iron/lead target were retained, respectively.

\section{RESULTS AND OUTLOOK}

Given the multi-dimensional aspect of this analysis, only a tiny fraction of the results can be shown here. The upper panels of Fig. 1 show the preliminary results for the acceptance and radiatively-corrected cross sections in $\mathrm{Q}^{2}$ and $\mathrm{p}_{\pi}$ for events produced on the carbon target which contain a positive pion. The lower panels show the data/MC ratio for the upper plots along with similar comparisons for the other three targets.

Current efforts in the analysis involve the determination of systematic errors and choosing kinematic cuts defined in such a way that comparisons with models (other than those included in GENIE) can be done in a meaningful fashion. We anticipate releasing 

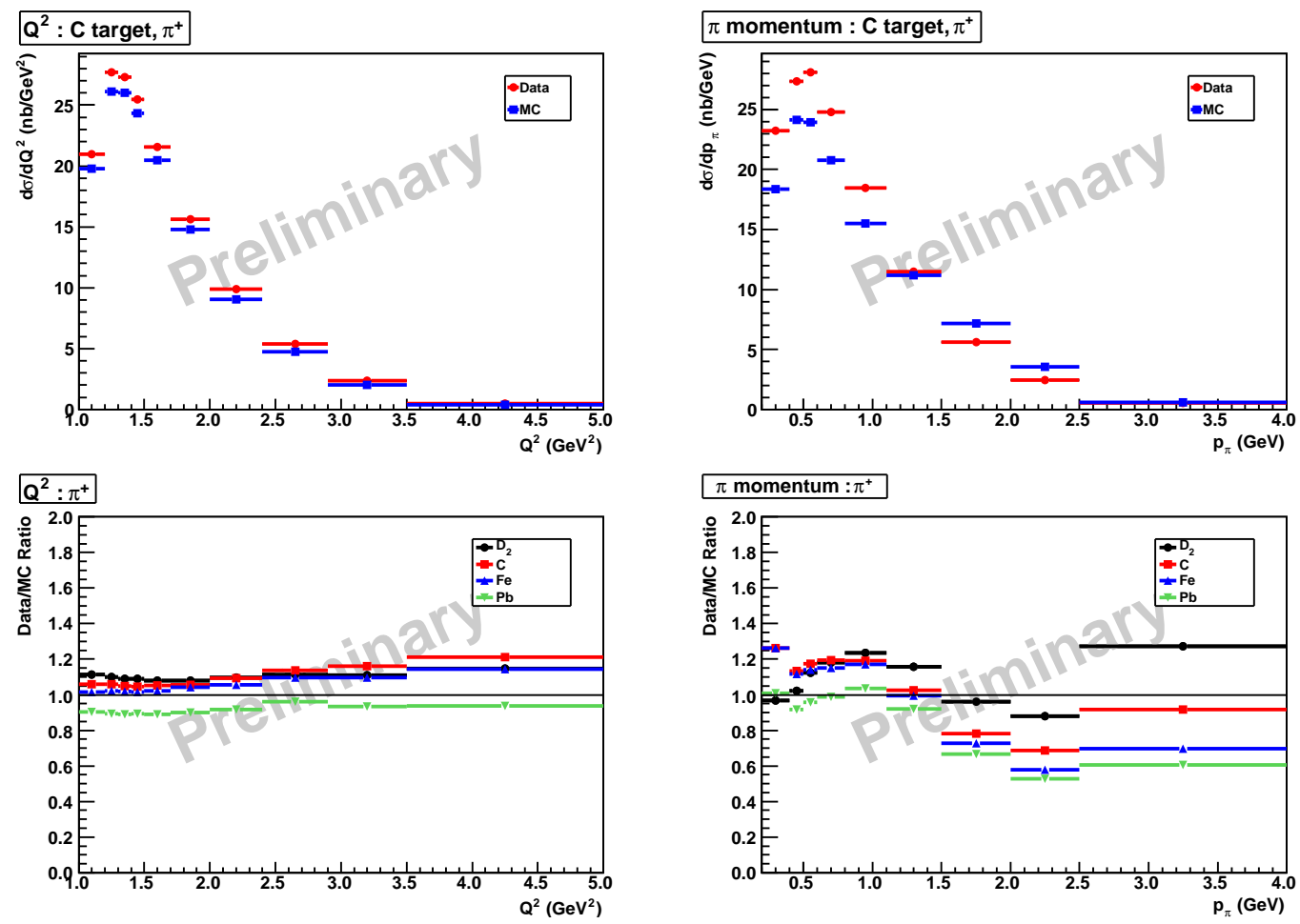

FIGURE 1. Representative distributions from this analysis.

final results in 2013 .

\section{REFERENCES}

1. See, for example, information on the T2K experiment, the MINOS experiment, and the NOvA experiment at http://www.t2k.org, http://www-numi.fnal.gov/, and http:// www-nova. fnal.gov/, respectively.

2. C. Andreopoulos, Electron scattering data and its use in constraining neutrino models, presented at the Sixth International Workshop on Neutrino-Nucleus Interactions in the Few-GeV Region, Sitges, Spain, May 2009.

3. O. Lalakulich, "Comparisons of theoretical calculations with MiniBooNE pion production data", presented at this conference, October 2012.

4. See http: //www. jlab.org.

5. B.A. Mecking et al., NIM A 503 (2003) 513.

6. The eg2 running period took data for JLAB experiments E02-104 and E02-110.

7. S. Manly, AIP Cof. Proc. 1405 (2011) 21-26.

8. H. Hakobyan et al., NIM A 592 (2008) 218.

9. P. Bosted, EG1-DVCS Technical Note \#005, April 2010.

10. See http: / /www . genie-mc . org/, Version 2.5.1.

11. D. Rein and L.M. Sehgal, Ann. Phys. 133 (1981) 79.

12. A. Bodek, U.K. Yang, J. Phys. G29 (2003) 1899.

13. C. Andreopoulos, Private communication, March 2011. 